\title{
Hydroelastic analysis of plates and some approximations
}

\author{
RODNEY EATOCK TAYLOR \\ Department of Engineering Science, University of Oxford, Parks Road, Oxford OX1 3PJ, UK \\ (e-mail:r.eatocktaylor@eng.ox.ac.uk)
}

\begin{abstract}
The paper concerns the modelling of very large pontoon-type floating structures by thin beams and plates of shallow draft, excited by regular waves. It is shown how the classical theory of hydroelasticity, involving the concepts of added mass and damping associated with the structural responses, may be reconciled with more recent formulations. In the latter, coupled equations for displacement and total hydrodynamic pressure are solved directly, without the breakdown into diffraction and radiation problems. A numerical model is adopted based on a Galerkin approach, and the nature of the various components of hydrodynamic loading on a shallow draft beam is investigated. The approach is then extended to the case of thin plate in waves, where the hydrodynamic effects are fully three dimensional.
\end{abstract}

Key words: hydroelasticity, waves, beam, plate

\section{Introduction}

I first encountered Nick Newman at the International Symposium on the Dynamics of Marine Vehicles and Structures in Waves, in 1974 at University College London. My interests then, and the paper I presented, concerned hydroelasticity [1, 2]. His paper [3] was on wave drift forces, and led to the widely used "Newman approximation" for low frequency drift forces. Subsequently he has published a number of papers on hydroelasticity $[4,5]$ (and I have had interests in drift forces). So it seemed appropriate that my contribution to this celebratory volume should return to the subject of hydroelasticity.

It is a subject which has attracted considerable interest in the last ten years, not least in the context of Very Large Floating Structures and the major activities related to the Megafloat project in Japan. This has encouraged the attention of hydrodynamicists around the world, who have been intrigued by the fascinating interaction of water waves and elastic waves in plates. The size of the structures, as envisaged for example for floating airports and spanning many wavelengths, poses substantial challenges in the area of numerical analysis. Several groups have contributed to much progress having been made in development of the tools for assessment of complex designs. Other have provided understanding of the underlying hydroelastic phenomena by applying advanced mathematical techniques to simple archetypes such as beams and semi-infinite plates. This paper lies somewhere between those two approaches, and attempts through some relatively simple analysis to bring together the modelling of beams and plates in order to shed a little light on the characteristics of certain mathematical models and the influence of three dimensional effects.

Most of the recent analyses of floating plates have been based on the procedure which formulates coupled equations for the deflection of the plate and the pressure on its underside. In some approaches these have been manipulated further to obtain a higher order differential 
equation for the deflection alone. The alternative classical procedure of separating the problem into diffraction and radiation components, as used universally for seakeeping analyses and extended for hydroelastic applications by Bishop and Price [6], is thereby avoided. The present paper considers how the two procedures relate to each other. It also examines some features of the two simple idealised models which have been extensively invoked to characterise the behaviour of very large floating platforms in waves, namely the free-free beam and the rectangular plate with free edges. While the restriction to behaviour in two dimensions is a plausible approximation for modelling the structural dynamics of a floating plate, it is not clear that the corresponding two dimensional hydrodynamic model will provide appropriate insight into the water wave interactions with a slender floating plate. In order to investigate this, a very simple numerical method is adopted here. We first consider the beam with two dimensional hydrodynamics, and then extend the method to the case of a rectangular plate and three dimensional hydrodynamics.

\section{Analysis of an elastic beam}

We adopt the formulation described by Khabakpasheva and Korobkin [7] and Eatock Taylor [8]. The governing equations are first summarised. The origin of the coordinate system $O x z$ is taken in the beam, with $z$ positive upwards. The water is of depth $d$. In the two dimensional problem the beam is assumed to have unit width. We non-dimensionalise the spatial coordinates by the length $L$ of the beam, and time by $\sqrt{L / g}$, where $g$ is the acceleration due to gravity. The dimensionless water depth is $\delta=d / L$. A sinusoidal wave of amplitude $A_{I}$ and non-dimensional wave number $k$ is assumed to be incident on the beam, propagating from right to left, and the complex amplitude of the deflection of the beam (nondimensionalised by $A_{I}$ ) is $w$. The hydrodynamic pressure on the underside of the beam has complex amplitude $p$, non-dimensionalised by $A_{I} \rho g$, where $\rho$ is the density of the fluid.

From the equation of motion of the beam, based on Euler-Bernoulli theory, we find that $w$ satisfies:

$$
\beta^{4} \frac{d^{4} w}{d x^{4}}-\mu K w=p
$$

where $K$ is the non-dimensional wave number in infinitely deep water given by the dispersion equation $K=k \operatorname{tank} k \delta$; and for a uniform beam of flexural rigidity $E I$ and draft $h$ we have

$$
\beta^{4}=\frac{E I}{\rho g L^{4}}, \mu=\frac{h}{L}
$$

We ignore structural damping. As shown in [7], a second equation may be obtained by writing Bernoulli's equation for the pressure $p$ in terms of a velocity potential $\phi$ and $w$. Thus

$$
\frac{p}{\rho}=-i \omega \phi-g w \text { on } z=0,
$$

where $\omega$ is the wave frequency. The draft $h$ is assumed to be small, so that this pressure on the underside of the plate is taken to be at $z=0$. The potential includes the incident wave and 
scattered wave components, and the latter may be expressed as an integral through use of Green's identity with a Green function $G\left(x, x_{o}\right)$. The result is

$$
p(x)+K \int_{0}^{1} G\left(x, x_{0}\right) p\left(x_{0}\right) d x_{0}=e^{i k x}-w(x)
$$

Solution of the coupled equations (1) and (3) yields the hydroelastic behaviour of the beam. More complex cases of non-uniform beams, or beams with hinges, may readily be analysed using a similar formulation. The approach is very convenient. It appears however to be rather different from the classical hydroelastic formulation [e.g. 6], in which the hydrodynamics are written in terms of diffraction and radiation problems, with appropriate definitions of added mass and added damping linked to some specified generalised coordinates. One of the aims of this paper is to shown how these two approaches are linked.

First we review the simple numerical method used here to obtain a selection of results. It is described at more length in [8], being based on a Galerkin formulation with the same assumed functions to represent the deflection $w$ and the pressure $p$. These are the rigid body modes of the beam and a finite set of sinusoidal functions, which were shown in [9] to be very convenient for representing the dynamics of a free-free beam. Thus we take

$$
w(x)=\sum_{n=0}^{N} w_{n} \psi_{n}(x), \quad p(x)=\sum_{n=0}^{N} p_{n} \psi_{n}(x),
$$

where

$$
\begin{aligned}
& \psi_{0}(x)=1 ; \quad \psi_{1}(x)=1-2 x \\
& \psi_{n+1}(x)=\sin n \pi x, \quad n \geq 1 .
\end{aligned}
$$

Note that the same number of terms is used in the series for $w$ and $p$.

These are now substituted into (1) and (3), each is multiplied by $\psi_{m}(x)$, and integrated over the length of the beam. Using the "weak" formulation of the Galerkin method, we integrate by parts twice, and equate the resulting weighted residuals to zero. The boundary terms at $x=-1,1$ resulting from these integrations by parts are zero. The final result, from [8], is

$$
\left[\begin{array}{cc}
-\boldsymbol{B}+K \mu \boldsymbol{A} & \boldsymbol{A} \\
\boldsymbol{A} & \boldsymbol{A}+K \boldsymbol{D}
\end{array}\right]\left[\begin{array}{l}
\boldsymbol{W} \\
\boldsymbol{P}
\end{array}\right]=\left[\begin{array}{l}
\boldsymbol{0} \\
\boldsymbol{P}^{I}
\end{array}\right]
$$

where the vectors $\boldsymbol{W}$ and $\boldsymbol{P}$ represent the unknown coefficients $w_{n}$ and $p_{n}$, and the terms of the other matrices and vector $\boldsymbol{P}^{I}$ are defined as follows:

$$
B_{m n}=\beta^{4} \int_{0}^{1} \psi_{m}^{\prime \prime} \psi_{n}^{\prime \prime} d x ; \quad A_{m n}=\int_{0}^{1} \psi_{m} \psi_{n} d x
$$




$$
D_{m n}=\int_{0}^{1} \int_{0}^{1} \psi_{m} \psi_{n} G d x d x_{0} ; \quad P_{m}^{I}=\int_{0}^{1} e^{i k x} \psi_{m} d x .
$$

$\boldsymbol{P}_{\boldsymbol{I}}$ corresponds to the pressure in the incident wave, the Froude-Krylov term.

The Green function $G$ is given [7] by:

$$
G\left(x, x_{0}\right)=\frac{-i k e^{-i k|x-x 0|}}{\delta\left(k^{2}-k^{2}\right)+K}+\sum_{j=1}^{\infty} \frac{s_{j} e^{-s_{j}\left|x-x_{0}\right|}}{\delta\left(s_{j}^{2}+k^{2}\right)-K},
$$

where $s_{j}$ are the imaginary roots of the dispersion equation. Apart from the need to truncate the series in (8), all the integrals in (7) may be obtained in closed form [8].

Solution of the linear algebraic system in (6), and substitution of $w_{n}$ and $p_{n}$ into the assumed forms (4), yields the required approximations for the deflection of the beam $w(x)$ and the total hydrodynamic pressure $p(x)$. The solution has been obtained without any consideration of separate diffraction and radiation problems, and indeed it is not immediately evident from the system of equations (6) where the hydrostatic effect is included. We now consider some special cases which together yield insight into how terms such as added mass and damping feature in the above formulation.

We consider three sub-problems. In problem I we examine response to an imposed external force in the absence of waves. Such forcing would provide an additional term on the right hand side of (1), which would lead to a non-zero vector, say $\boldsymbol{F}$, replacing the vector of zeroes on the right hand side of (6). Thus for a distributed force $f(x)$, we have the terms

$$
F_{m}=\int_{0}^{1} f(x) \psi_{m}(x)
$$

We may then eliminate $\boldsymbol{P}$ using the second set of equations in (6), and obtain the response to the external forcing as the formal solution of

$$
[-\boldsymbol{B}+K \mu \boldsymbol{A}+\boldsymbol{A H}] \boldsymbol{W}=\boldsymbol{F}
$$

where

$$
\boldsymbol{H}=-(\boldsymbol{A}+K \boldsymbol{D})^{-1} \boldsymbol{A} .
$$

In a similar manner, in problem II we obtain the force on the beam when it is oscillated (again in the absence of incident waves) by a prescribed displacement. With a model approximated by say $(N+1)$ coefficients $w_{n}$ in (4), we may solve $(N+1)$ problems, each defined by the imposition of a unit value of $w_{n}$ with all the other coefficients of the series for $w(x)$ set to zero. Let us define the $n^{\text {th }}$ column of a matrix $\boldsymbol{Q}$ as the solution to the coefficients of the series for $p(x)$ corresponding to the imposed displacement $w(x)=\psi_{n}(x)$. Then, from (6), we find 


$$
\boldsymbol{Q}=\boldsymbol{H},
$$

using the definition in (11).

Consider now the hydrodynamic loading on the beam when it responds to external forcing according to (10). This corresponds to the hydrodynamic pressure $p$ in (1), which in the discretised form of (10) is represented by the term $\boldsymbol{A H W}$ : this is precisely the term $\boldsymbol{A Q W}$ obtained in problem II. It must therefore correspond to both radiation terms (added mass and damping) and the hydrostatic restoring force. The latter, following from (2), is $-\boldsymbol{A} \boldsymbol{W}$. Hence the radiation term is given by $\boldsymbol{A}(\boldsymbol{Q}+\boldsymbol{I}) \boldsymbol{W}$ where $\boldsymbol{I}$ is the identity matrix. Expressed in the classical form, therefore, (10) would be written as:

$$
\begin{aligned}
& B W-K \mu A W-A(Q+I) W+A W=F . \\
& \text { elastic }+ \text { inertia }+ \text { radiation }+ \text { hydrostatic }=\text { external } \\
& \text { stiffness term force force force }
\end{aligned}
$$

Correspondingly, the approximated radiation force $p^{R}$ would be expressed as:

$$
p^{R}(x)=\sum_{m=0}^{N} \psi_{m}(x) \sum_{n=0}^{N}\left(Q_{m n}+1\right) w_{n}
$$

analogous to the approximation for $p$ in (4).

For problem III, diffraction by a fixed beam, we proceed in a similar manner. We set $\boldsymbol{W}=\boldsymbol{0}$ in (6) and solve for the total diffraction pressure term, $\boldsymbol{P}^{\boldsymbol{D}}$, obtaining:

$$
\boldsymbol{P}^{\boldsymbol{D}}=(\boldsymbol{A}+K \boldsymbol{D})^{-1} \boldsymbol{P}^{\boldsymbol{I}}
$$

Note that $\boldsymbol{P}^{\boldsymbol{D}}$ includes the effect of both incident and diffracted waves: it arises from the total pressure in (2) - in this case with $w=0$.

Finally, using the above results, we may rewrite the solution for the floating plate in waves. From the second part of (6), and using (15), we have

$$
(\mathbf{A}+K \boldsymbol{D})^{-1} \mathbf{A W}+\boldsymbol{P}=\boldsymbol{P}^{D} .
$$

Inserting (11) and (12) in (16) and transferring terms from one side to the other side of the equation, we obtain:

$$
\boldsymbol{P}=\boldsymbol{P}^{D}+(\boldsymbol{Q}+\boldsymbol{I}) \boldsymbol{W}-\boldsymbol{W} .
$$

Hence after changing the signs of each term, the first part of (6) can be written in the form:

$$
\begin{aligned}
& B W-K \mu A W-A(Q+I) W+A W=A P^{D} . \\
& \text { elastic }+ \text { inertia }+ \text { radiation }+ \text { hydrostatic }=\text { wave } \\
& \text { stiffness term force force force }
\end{aligned}
$$




\section{Beam results}

Using the above-mentioned methodology, results have been obtained for a case considered by Wu et al. [10]. They have shown comparisons between experimental results for a beam spanning across the width of a long flume, and a numerical approach based on the eigenfunction expansion matching technique. Broadly speaking the experimental data confirmed their numerical approach, based on using up to 30 terms of the eigenfunction series. Subsequent work [11] showed that at least 50 terms were required to obtain converged results to plotting accuracy for the cases considered in [10]. Those converged results are compared in Figure 1 with those calculated using the theory of the previous section, with different numbers $N$ of sinusoidal functions. The results correspond to a beam with the nondimensional parameters $\delta=0.11, \mu=0.836 \times 10^{-3}, \beta_{\text {ref }}=4.8025 \times 10^{-6}$, and with the series for the Green function truncated at 45 terms. The modulus of the displacement of the beam, $|w|$, is shown in Figure 1(a) and 1(b) respectively for two wave periods, corresponding to nondimensional wave numbers $K=19.71$ and $K=4.870$. It is seen that for these cases $N=20$ provides satisfactory agreement in the graphs with the converged results based on [11].

(a)

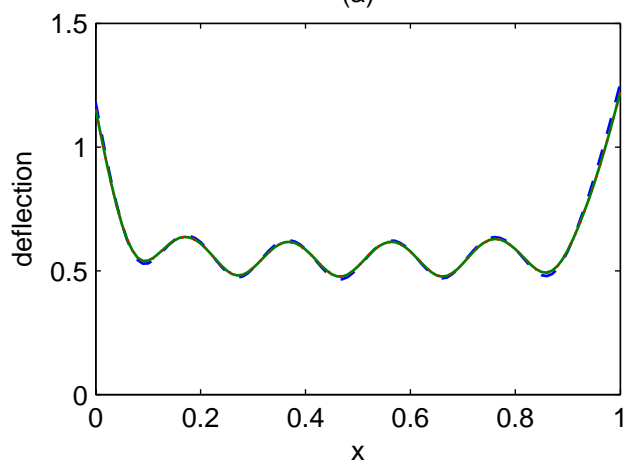

(b)

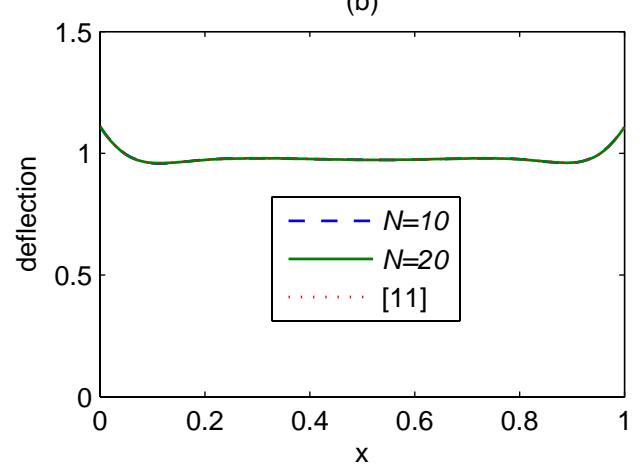

Figure 1 Modulus of the deflection of a beam, from [11] and calculated with different numerical models. (a) $K=19.71$; (b) $K=4.870$.

Next we consider the breakdown of the hydrodynamic force into diffraction, radiation, and hydrostatic terms, as discussed above. Figure 2 shows the real parts (lower three lines) and imaginary parts (upper three lines) of the wave forces (incident plus diffracted) for the same beam in the wave corresponding to $K=19.71$, for the non-dimensional water depths $\delta=0.11$, $\delta=0.0731$ and $\delta=0.3188$. These are equivalent to depth to wave length ratios in the range 0.25 to 0.5 , the latter being effectively infinite depth. A notable feature of these results is that the wave force does not exhibit oscillations corresponding to the incident wave length (for $\delta$ $=0.11$, the dimensionless value of the wave length is 0.311 ). The effect of diffraction by this shallow draft beam roughly cancels the incident wave at its downwave end (the left hand side of the figure); the magnitude of the total diffraction force increases almost monotonically towards a maximum amplitude at the upwave end. 


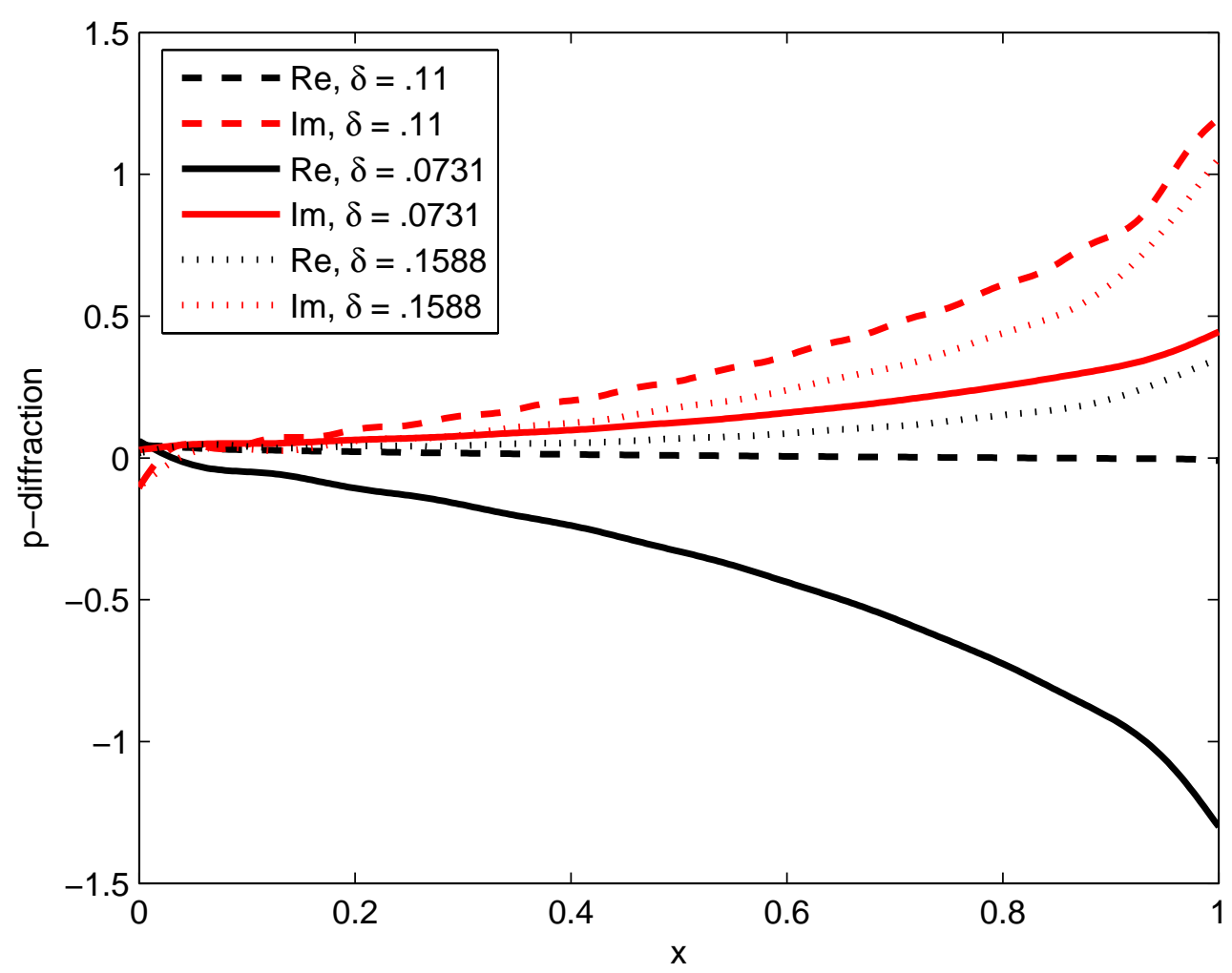

Figure 2 Real and imaginary parts of diffraction force on a beam, in three water depths. (a) $\delta=0.11$; (b) $\delta=0.0731$; (c) $\delta=0.3188$.

Figure 3 shows the real and imaginary parts of the hydrostatic, radiation and total hydrodynamic forces for the case considered in [10] and Figure 1, again for $\mathrm{K}=19.71$. Corresponding results are illustrated for a more flexible beam with $\beta=10^{-3} \beta_{\text {ref }}$ in Figure 4, and for a stiffer beam, $\beta=10^{3} \beta_{\text {ref }}$, in Figure 5 . The negative of the hydrostatic force distribution, the left hand frame in each case, is the deflected shape, so it is unnecessary to plot the latter separately. The total force, the right hand plot in each case, has been calculated in two ways: it is given directly by the series for $p$ given in (4); and it is obtained from the sum of the diffraction, radiation and hydrostatic forces shown in the other figures. The graphical results are seen to be the same from the two approaches. These figures confirm the major influence on the responses of the beam of its elastic properties over this range. For the very flexible case shown in Figure 4, the beam essentially follows the incident wave. At the downwave end, where the diffraction force is seen in Figure 2 to tend to zero, the radiation force is roughly $180^{\circ}$ out of phase with the hydrostatic force (implying low hydrodynamic damping). The total hydrodynamic force is seen to be very small along the whole length of the beam. The cancellation effects seen here suggest the importance of accurately calculating the separate components, if they are evaluated independently. 

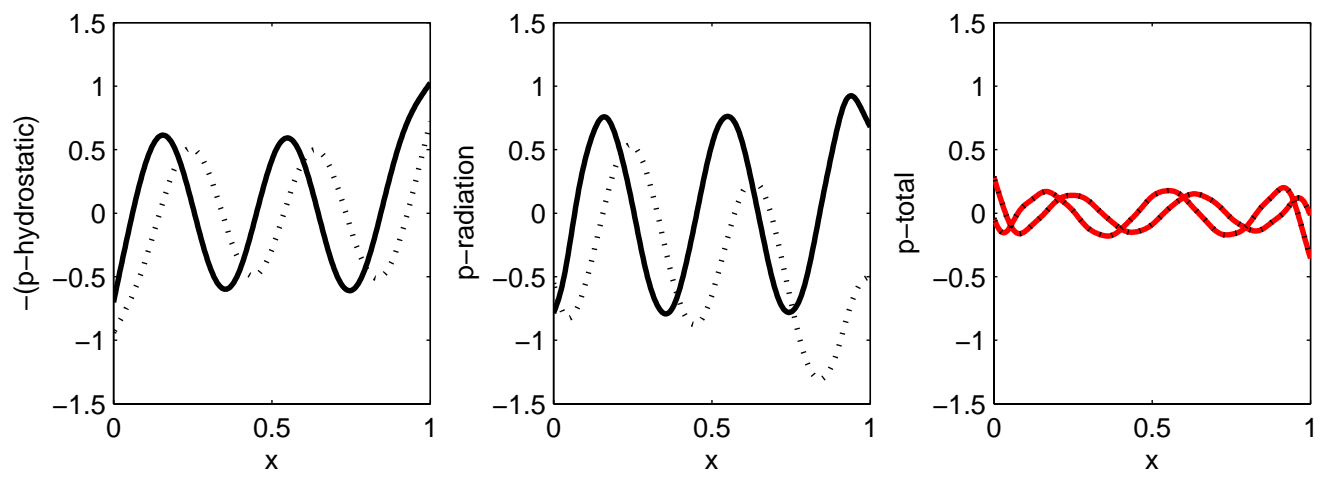

Figure 3 Real and imaginary parts of hydrostatic, radiation and total hydrodynamic force on the beam of flexibility $\beta_{r e f}$, for $K=19.71$.
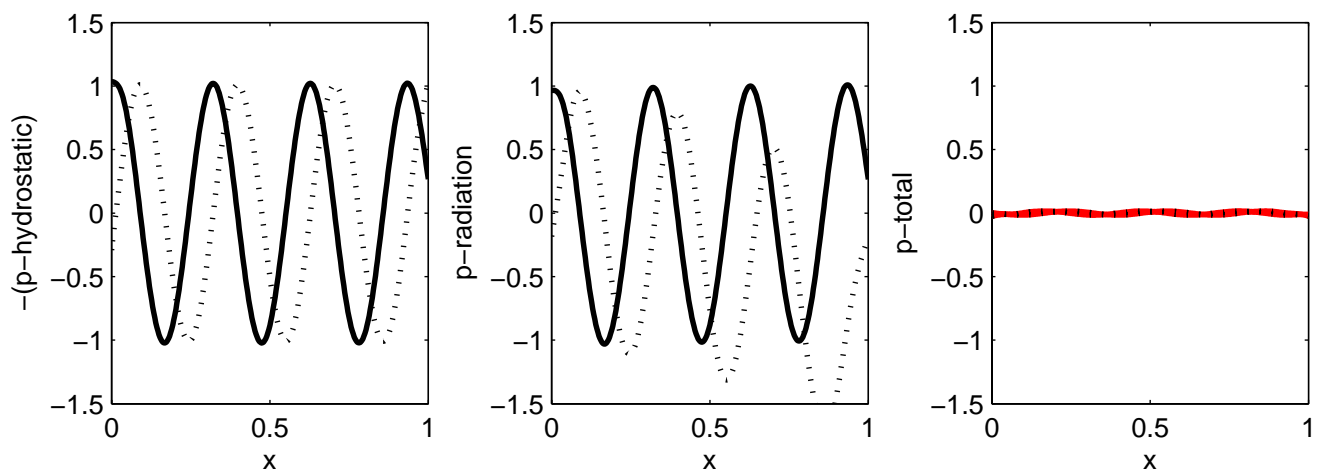

Figure 4 As for Figure 3, but with $\beta=10^{-3} \beta_{\text {ref }}$.
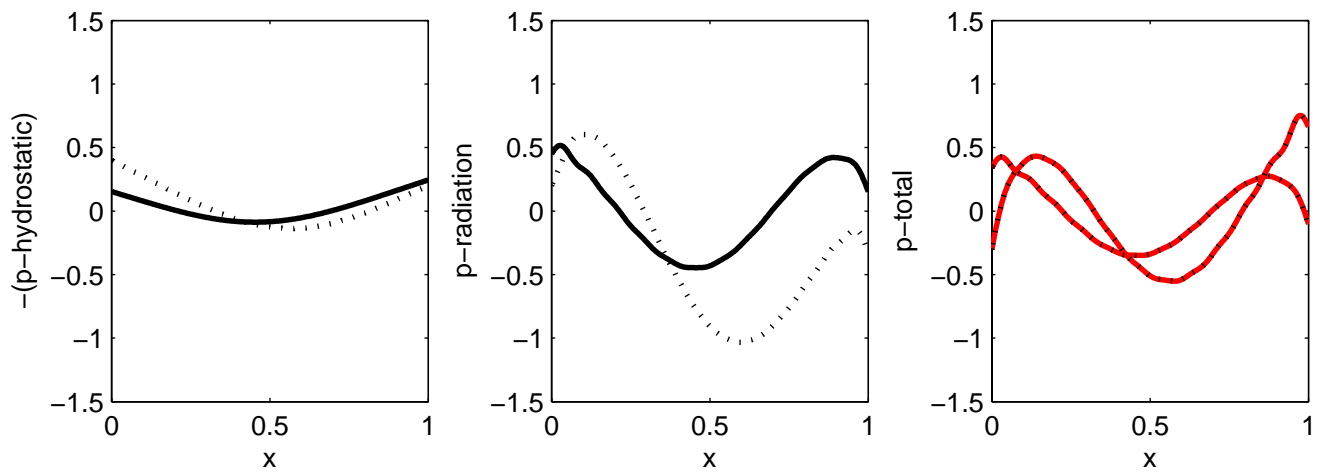

Figure 5 As for Figure 3, but with $\beta=10^{3} \beta_{\text {ref }}$.

\section{The plate model}

We now extend the previous analysis to the case of a rectangular plate of length $L$ and width $B$. We will here make the simplification that the plate is rigid in relation to bending about axes parallel to the (long) sides of length $L$. The flexural rigidity is taken to be $E I$ per unit width of the plate about axes parallel to the (short) sides. The (small) draft is again $h$. The 
coordinate system $\mathrm{O} x y z$ has origin $\mathrm{O}$ at the centre of the plate, with $\mathrm{O} x$ directed parallel to the long side and $\mathrm{O} z$ measured positive upwards from the water plane area.

The equation governing the motions of the plate, analogous to (1), is:

$$
\beta^{4} \frac{d^{4} w}{d x^{4}}-\mu K w=\int_{-1}^{1} p(x, y) d y
$$

where now the coordinates $x$ and $y$ are non-dimensionalised by $L / 2$ and $B / 2$ respectively.

Subsequently we use an aspect ratio defined by $b=B / L$. The pressure is here non-

dimensionalised by $A_{I} \rho g B$, and the definitions of $\beta^{4}$ and $\mu$ are the same as those given after (1). The non-dimensional wave number is now $K=k L / 2$.

Proceeding as for the beam, we obtain

$$
\begin{gathered}
p(x, y)+K \int_{-1}^{1} \int_{-1}^{1} G\left(x, y ; x_{0}, y_{0}\right) p\left(x_{0}, y_{0}\right) d x_{0} d y_{0} \\
=e^{-i K x}-w .
\end{gathered}
$$

Here $G$ is the three dimensional Green function for points on the free surface, given [12] by

$$
G\left(x, y ; x_{o}, y_{o}\right)=\frac{-K}{4 \pi}\left\{\frac{2}{z}-\pi\left[\mathrm{H}_{0}(z)+\mathrm{Y}_{0}(z)+2 \pi \mathrm{J}_{0}(z)\right]\right\}
$$

where $z=K\left[\left(x-x_{0}\right)^{2}+\left(y-y_{0}\right)^{2}\right]^{1 / 2}$. The functions $\mathrm{H}_{0}, \mathrm{Y}_{0}$ and $\mathrm{J}_{0}$ are the zeroth order Struve function and the zeroth order Bessel functions of the first and second kind respectively.

Equations (19) and (20) are the coupled equations for the plate deflection $w$ and pressure $p$, which we again solve by the Galerkin method. We use assumed functions in the $x$ and $y$ directions as follows:

$$
w(x)=\sum_{m=0}^{M} w_{m} \psi_{m}(x), \quad p(x, y)=\sum_{m=0}^{M} \sum_{n=0}^{N} p_{m n} \psi_{m}(x) \chi_{n}(y)
$$

where

$$
\begin{aligned}
& \psi_{0}(x)=1=\chi_{0}(y), \psi_{1}(x)=x \\
& \psi_{m}(x)=\sin (m-1) \frac{\pi}{2}(1+x), m \geq 2 \\
& \chi_{n}(y)=\cos (2 n-1) \frac{\pi}{2} y, n \geq 1 .
\end{aligned}
$$


This choice is governed by the restriction here to responses of the plate in head seas.

As before, the representations of $w$ and $p$ in (23) are substituted into (19), the result multiplied by $\psi_{p}(x)$, and integrated over the length of the plate. The same substitution is made in (20), which is then multiplied by $\psi_{p}(x) \chi_{q}(y)$ and integrated over the length and width of the plate. The first of the resulting equations is then integrated by parts twice in the $x$ direction. This leads to the following coupled equations for $w_{m}$ and $p_{m n}$ :

$$
\begin{gathered}
\sum_{m=0}^{M}\left[\beta^{4} \int_{-1}^{1} \psi_{m}^{\prime \prime} \psi_{p}^{\prime \prime} d x-K \int_{-1}^{1} \psi_{m} \psi_{p} d x\right] w_{m}-\sum_{m=0}^{M} \sum_{n=0}^{N}\left[\int_{-1}^{1} \psi_{m} \psi_{p} d x \int_{-1}^{1} \chi_{n} d y\right] p_{m n}=0 \\
\sum_{m=0}^{M}\left[\int_{-1}^{1} \psi_{m} \psi_{p} d x \int_{-1}^{1} \chi_{q} d y\right] w_{m}+\sum_{m=0}^{M} \sum_{n=0}^{N}\left[\iint_{-1}^{1} \int_{-1}^{1} \psi_{m} \psi_{p} d x \int_{-1}^{1} \chi_{n} \chi_{q} d y\right. \\
\left.+b K \int_{-1}^{1} \int_{-1}^{1} \int_{-1}^{1} \int_{-1}^{1} G \psi_{m} \psi_{p} \chi_{n} \chi_{q} d x_{o} d y_{o} d x d y\right]=\int_{-1}^{1} \psi_{p} e^{-i k x} d x \int_{-1}^{1} \chi_{q} d y
\end{gathered}
$$

In matrix form these become:

$$
\left[\begin{array}{cc}
-\boldsymbol{C}+K \mu \boldsymbol{A} & \boldsymbol{R} \\
\boldsymbol{R}^{T} & \boldsymbol{U}+K \boldsymbol{D}
\end{array}\right]\left[\begin{array}{l}
\boldsymbol{W} \\
\boldsymbol{P}
\end{array}\right]=\left[\begin{array}{l}
\boldsymbol{0} \\
\boldsymbol{E}
\end{array}\right]
$$

This of course has a similar form to (6) for the two-dimensional problem above, but with the terms defined as follows:

$$
\begin{aligned}
& W_{m}=w_{m}, \quad P_{(n-1) M+m}=p_{m n}, \\
& C_{p m}=\beta^{4} \int_{-1}^{1} \psi_{m} \psi_{p} \psi_{p} d x \\
& A_{p m}=\int_{-1}^{1} \psi_{m} \psi_{p} d x, \\
& R_{p m^{\prime}}=\frac{1}{4} \int_{-1}^{1} \psi_{m} \psi_{p} d x \int_{-1}^{1} \chi_{n} d y, m^{\prime}=(n-1) M+m, \\
& U_{p^{\prime} m^{\prime}}=\frac{1}{4} \int^{1} \psi_{m} \psi_{p} d x \int_{-1}^{1} \chi_{n} \chi_{q} d y, p^{\prime}=(q-1) M+p, \\
& D_{p^{\prime} m^{\prime}}=\frac{b}{16} \int_{-1}^{1} \int_{-1}^{1} \int_{-1}^{1} \int_{-1}^{1} G \psi_{m} \psi_{p} \chi_{n} \chi_{q} d x_{0} d y_{0} d x d y,
\end{aligned}
$$




$$
P_{p^{\prime}}^{I}=\frac{1}{4} \int_{-1}^{1} e^{-i K x} \psi_{p} d x \int_{-1}^{1} \chi_{q} d y
$$

By solving the matrix equations (26) for any particular frequency $\omega$, it is then possible to obtain the plate deflection $w$ and the hydrodynamic pressure $p$. The accuracy of the solution will depend on the number of terms $M$ and $N$ in the assumed solution, and on the accuracy of the integrations. For the rectangular plate, all of the integrals may be obtained analytically, expect for that involving the Green function, which requires special treatment in view of the inherent singularity. This is discussed next.

\section{Numerical evaluation of the Green function integral}

The Green function $G(z)$ contains the singularity $1 / z$ and also, because of the second kind Bessel function $\mathrm{Y}_{0}(z)$, the logarithmic singularity $(2 / \pi) \ln z$. Fortunately, difficulties associated with these may be avoided through partial analytical integration. The two key results, for a point $(\xi, \eta)$ in the first quadrant, are:

$$
\begin{aligned}
I 2_{1}(x, y)=K \int_{\eta}^{1} \int_{x}^{1} \frac{d x_{0} d y_{0}}{z}= & (1-x) \ln \left[\frac{\alpha+b(1-y)}{1-x}\right]+b(1-y) \ln \left[\frac{\alpha+1-x}{b(1-y)}\right], \\
I 2_{2}(x, y)=\int_{\eta}^{1} \int_{x}^{1} \ln z d x_{o} d y_{o}= & \frac{1}{2}(1-x)^{2} \theta+\frac{1}{2} b^{2}\left(1-y^{2}\right)\left(\frac{\pi}{2}-\theta\right) \\
& -\frac{b}{2}(1-x)(1-y)[3-2 \ln (K \alpha)],
\end{aligned}
$$

where

$$
\alpha^{2}=(1-x)^{2}+b^{2}(1-y)^{2}, \quad \tan \theta=\frac{b(1-y)}{1-x}
$$

The quadruple integrals are obtained by integrating these with respect to $x$ and $y$ over the rectangle. For example, over a square $(b=1)$, we can obtain for the rigid body heave mode $(m=n=p=q=0)$ :

$$
\begin{aligned}
& I 4_{1}=K \int_{-1-1-1-1}^{1} \int^{1} \int^{1} \int_{-1}^{1} \frac{1}{z} d x_{o} d y_{o} d x d y=\int_{-1}^{1} \int_{-1}^{1} I 2_{1}(x, y) d x d y=23.78568, \\
& I 4_{2}=\int_{-1}^{1} \int_{-1}^{1} \int_{-1}^{1} \int_{-1}^{1} \ln z d x_{o} d y_{o} d x d y=\int_{-1}^{1} \int_{-1}^{1} I 2_{2}(x, y) d x d y=-1.791033+16 \ln K .
\end{aligned}
$$

The latter double integrals are obtained by straightforward numerical integration using the mathematical package Mathcad.

More generally, we may use $I 2_{1}$, and $I 2_{2}$ to deal with the singularities in the expression for $D_{p^{\prime} m}$ ' in (27), involving the frequency dependent Green function multiplied by the basis functions. We use: 


$$
\begin{aligned}
I_{\text {mnpq }}= & \int_{-1}^{1} \int_{-1}^{1} \int_{-1}^{1} \int_{-1}^{1} G \psi_{m} \psi_{p} \chi_{n} \chi_{q} d x_{0} d y_{0} d x d y= \\
& \frac{-1}{2 \pi} \int_{-1}^{1} \int_{-1}^{1} 4\left[I 2_{1}(x, y)-K I 2_{2}(x, y)\right] \psi_{m}(x) \chi_{n}(y) \psi_{p}(x) \chi_{q}(y) d x d y \\
& -K \int_{-1}^{1} \int_{-1}^{1} \int_{-1-1}^{1} \int_{-1}^{1}\left\{\frac{1}{2 \pi}\left[\frac{1}{z}-\ln z\right]\left[\psi_{m}\left(x_{0}\right) \chi_{n}\left(y_{0}\right)-\psi_{m}(x) \chi_{n}(y)\right]\right. \\
& \left.-\frac{1}{4}\left[\mathrm{H}_{0}(z)+\left(\mathrm{Y}_{0}(z)-\frac{2}{\pi} \ln z\right)+2 \pi i \mathrm{~J}_{0}(z)\right] \psi_{n}\left(x_{0}\right) \chi_{n}\left(y_{0}\right)\right\} \psi_{p}(x) \chi_{q}(y) d x_{0} d y_{0} d x d y .
\end{aligned}
$$

The fourfold integrals still have to be evaluated numerically, for which it is convenient to use high order Gaussian quadrature. The coordinates of the integration points and the weights are calculated using the algorithm given by Recktenwald [13]. By choosing the integration order $N_{G}$ in one direction to be different from that in the other (e.g. $N_{G}$ and $N_{G}$-2 where $N_{G}$ is even), we may avoid coincidence of the source and field points in the Green function (as suggested in [12]).

\section{Plate results}

First the numerical approach for dealing with the integrals has been checked by making a comparison with some results by Campbell [14] for flexible diaphragms. The integral I4 corresponds to the added mass of a rigid diaphragm oscillating in a direction perpendicular to an infinite rigid plane (the "closed edge" case described by Campbell). He evaluated the added masses for a range of deflected shapes of diaphragms using a panel method (with constant panels). Several aspect ratios were calculated, and he then provided a parametric fit to allow results to be estimated for any aspect ratio. Thus for a plate with side lengths $L \times B$, the added mass, $M$, corresponding to deflection in some prescribed mode is written by Campbell in a form equivalent to:

$$
M=\rho L B^{2} \gamma\left(1+\alpha_{1} \tau+\alpha_{2} \tau^{2}+\alpha_{3} \tau^{3}\right)
$$

where $\tau=\ln (L / B)$. Here we have used a similar parametric fit (for two of the cases), but calculated the added mass for each geometry and oscillation mode $\psi(x)$ using the simple methods described above. The first, (i), uses the analytical result for the double integral $I 2_{1}$ in (28) followed by a numerical integration in Mathcad; while the second, (ii), uses Gaussian integration to perform the fourfold integral, with different numbers of integration points. The results are compared with those of Campbell [14] in Table 1. The results from [14] are designated (iii). Three cases are shown, corresponding to a rigid body displacement of the plate, a parabolic distortion in one direction, and distortion of a square plate $(\tau=0)$ in the ninth sinusoidal mode. The approach represented by (30) was used to evaluate the integral in the latter cases. Reasonable agreement is found with the published results, and satisfactory convergence. 
Table 1 Parameters for added masses of a rectangular diaphragm: (i) semi-analytical; (ii) by Gauss quadrature (with different numbers of integration points), (iii) from Campbell [14].

\begin{tabular}{|c|c|c|c|c|c|c|}
\hline$\psi(x)$ & $\chi(y)$ & Method - Gauss points & $\gamma$ & $\alpha_{1}$ & $\alpha_{2}$ & $\alpha_{3}$ \\
\hline 1 & 1 & $\begin{array}{c}\text { (i) } \\
\text { (ii) }-64 \\
\text { (ii) }-32 \\
\text { (ii) }-16 \\
\text { (iii) }\end{array}$ & $\begin{array}{c}0.9464 \\
0.9462 \\
0.9455 \\
0.9420 \\
0.944 \\
\end{array}$ & $\begin{array}{l}0.5024 \\
0.5022\end{array}$ & $\begin{array}{l}0.0596 \\
0.0597\end{array}$ & $\begin{array}{l}-0.0081 \\
-0.0083\end{array}$ \\
\hline $1-x^{2}$ & 1 & $\begin{array}{l}\text { (ii) }-64 \\
\text { (ii) }-32 \\
\text { (ii) }-16 \\
\text { (iii) }\end{array}$ & $\begin{array}{c}0.4762 \\
0.4777 \\
0.4789 \\
0.473\end{array}$ & 0.471 & 0.044 & -0.006 \\
\hline $\sin \frac{9 \pi}{2}(1+x)$ & 1 & $\begin{array}{l}\text { (ii) }-64 \\
\text { (ii) }-32 \\
\text { (ii) }-16\end{array}$ & $\begin{array}{l}1.326 \\
1.332 \\
1.366\end{array}$ & & & \\
\hline
\end{tabular}

Next we consider a selection of results for waves incident on a square plate, and on a rectangular plate of aspect ratio 0.2 , in water of infinite depth. We take $\mu=1.67 \times 10^{-3}$ and for the base case $\beta_{\text {ref }}=0.0916 \times 10^{-3}$. We also consider stiffer plates of the same geometries, for which $\beta=10^{3} \beta_{\text {ref. }}$. The rectangular plate having the latter stiffness is effectively the same as that considered by Kashiwagi [15], though in that case the plate was in shallow water depth. In all cases the wave frequency corresponds to $K=6.273$, which gives a wavelength about half the length of the plate.

Figure 6 shows results for the square plate, on the left showing the reference case, and on the right the stiffer plate. In each case 5 lines are plotted, representing the results obtained from different numerical models. Each is identified by the code $n_{1} n_{2} n_{3}$, where $n_{1}$ is the number of Gaussian points in the $x$ direction; $n_{2}$ is the number of functions in the $x$ direction; and $n_{3}$ is the corresponding number in the $y$ direction (these are one more than the numbers $M$ and $N$ in equation (22)). It may be seen that increasing $n_{1}$ from 32 to 64 has only a small effect on the results. As can be expected, convergence with respect to the number of functions becomes quite sensitive at the ends of the plate.

Figure 7 shows the equivalent results for the rectangular plate. Again the increase in the number of Gauss points does not change the results much for the parameters examined. A larger number of functions in the $y$ direction, however, is now seen to be necessary to achieve comparable accuracy. There is little difference between the plots for cases 321206, 321606 and 321608. 

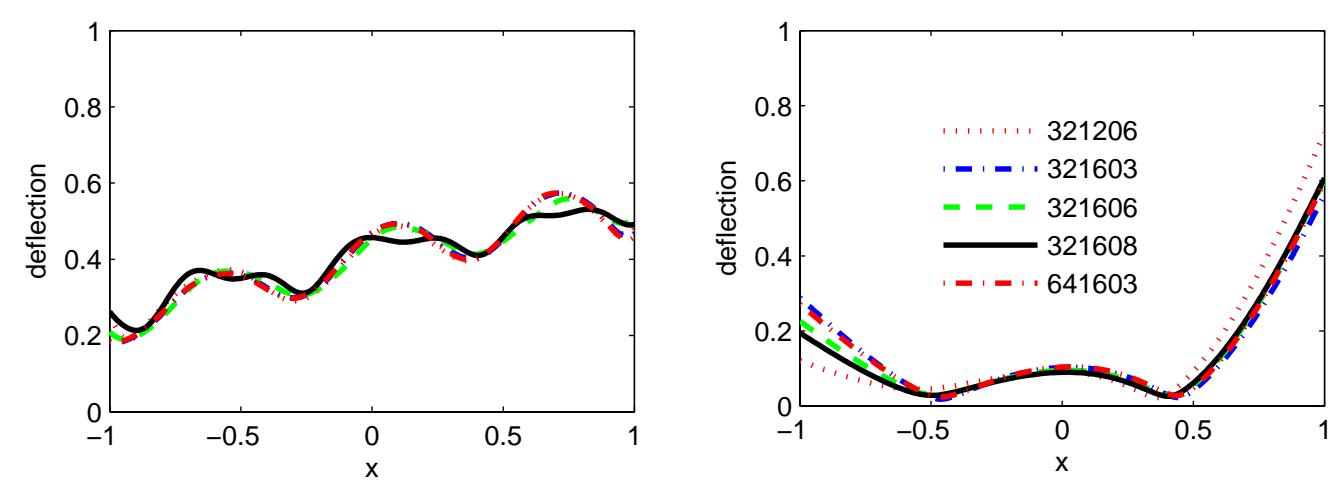

Figure 6 Modulus of deflection of square plate, calculated with different numerical models.
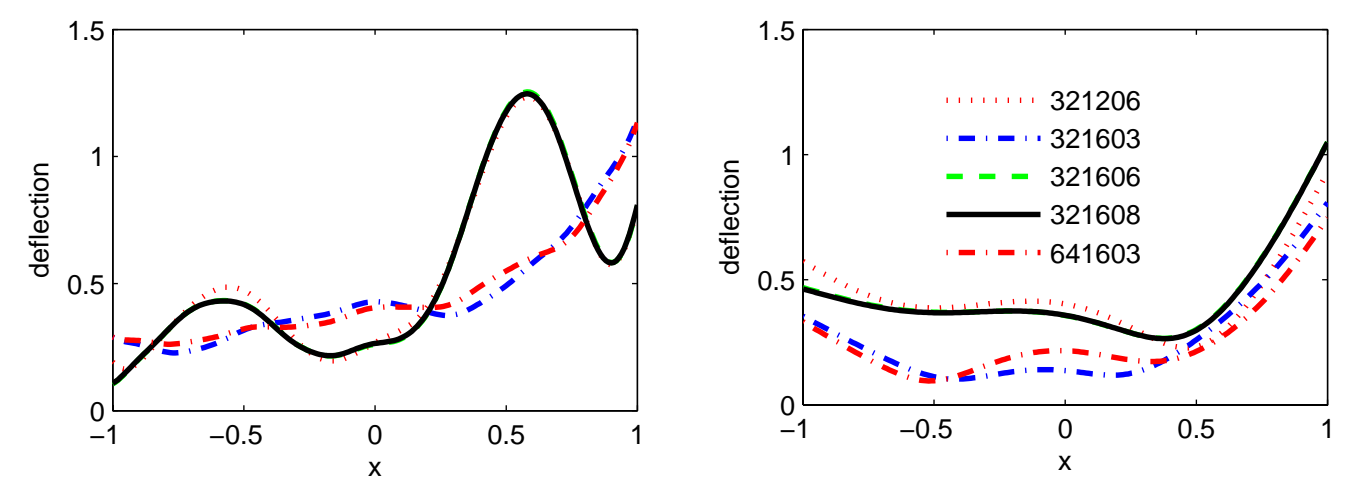

Figure 7 Modulus of deflection of rectangular plate of aspect ratio 0.2, calculated with different numerical models.

\section{Concluding remarks}

The origin of this work was an attempt to shed some light on two facets of recent analyses of the hydroelastic behaviour of very thin plates in waves. The first related to a reconciliation between two mathematical models which have been developed over the last 30 years: one model poses the problem in terms of a decomposition of the hydrodynamic behaviour into diffraction, radiation and hydrostatic components; but it appears that this approach has not been much (if at all) used for the very thin plate problem. The other approach couples the plate deflection and the total hydrodynamic pressure, with no explicit representation of effects such as added mass and damping. In this paper we have used the second approach, but shown how the diffraction and radiation terms may in fact be extracted from the coupled equations. This has been illustrated for the case of a beam, using a simple scheme based on a Galerkin approximation to obtain the numerical results. In the cases considered, the separate diffraction, radiation and hydrostatic components are all much larger in magnitude than their combined effect.

The other facet of interest is the relationship between two and three dimensional analyses for the floating plate problem. It can be argued that the simple model of a beam provides some insight into the complex hydroelastic phenomena exhibited by a long rectangular plate. But if the associated hydrodynamic analysis is two-dimensional, the resulting response may be very different from what would be predicted with a model in which three dimensional hydrodynamic effects can be represented. This has been shown here by comparing results 
from square and rectangular plates under the same conditions. It is clear that the overall response of the floating plate is extremely sensitive to its aspect ratio. But the limiting case where the plate width is extended to approach a case equivalent to the beam model has not been attempted, because based on the very simple numerical method described here the number of terms required to provide an adequate representation of the hydrodynamic behaviour in the transverse direction would appear to be excessive. A more sophisticated approach could resolve that issue.

\section{References}

1. R. Eatock Taylor, Analysis of the flexural vibrations of variable density spheroids immersed in an ideal fluid, with application to ship structural dynamics. Phil. Trans. $R$. Soc. Lond. A277 (1975) 623-648.

2. R. Eatock Taylor, Structural dynamics of fixed and floating platforms in waves. In: R.E.D. Bishop and W.G. Price (eds.), The Dynamics of Marine Vehicles and Structures in Waves. London: The Institution of Mechanical Engineers (1975) pp. 235-247.

3. J.N. Newman, Second order, slowly varying forces on vessels in irregular waves. In: R.E.D. Bishop and W.G. Price (eds.), The Dynamics of Marine Vehicles and Structures in Waves. London: The Institution of Mechanical Engineers (1975) pp.182-186.

4. J.N. Newman, Wave effects on deformable bodies. Appl. Ocean Res. 16 (1994) 47-59.

5. C.-H. Lee and J.N. Newman, An assessment of hydroelasticity for very large hinged vessels. J. Fluids Struct. 14 (2000) 957-970.

6. R.E. D. Bishop and W.G. Price, Hydroelasticity of Ships. Cambridge: Cambridge University Press (1979) 423 pp.

7. T.I. Khabakhpasheva and A.A. Korobkin, Hydroelastic behaviour of compound floating plate in waves. J. Engng. Math. 44 (2002) 21-40.

8. R. Eatock Taylor, Wet or dry modes in linear hydroelasticity - why modes? In: R. Eatock Taylor (ed.), Proceedings of $3^{\text {rd }}$ International Conference on Hydroelasticity in Marine Technology. Oxford: The University of Oxford (2003) pp. 239-250.

9. R. Eatock Taylor and M. Ohkusu, Green functions for hydroelastic analysis of vibrating free-free beams and plates. Appl. Ocean Res. 22 (2000) 295-314.

10. C. Wu, E. Watanabe and T. Utsunomiya, An eigenfunction expansion matching method for analysing the wave-induced responses of an elastic floating plate. Appl. Ocean Res. 17 (1995) 301-310.

11. T. Utsunomiya, Private communication (2003).

12. C.D. Wang and M.H. Meylan, A higher-order coupled boundary element and finite element method for wave forcing of a floating elastic plate. J. Fluids Struct. 19 (2004) 557-572. 
13. G. Recktenwald, Numerical Methods with MATLAB: Implementations and Applications. Upper Saddle River, N.J.: Prentice Hall (2000) 786pp.

14. W.F. Campbell, The Added Mass of Some Rectangular and Circular Flat Plates and Diaphragms. Report LTR-LA-223. Ottawa: National Research Council of Canada (1982) 45pp.

15. M. Kashiwagi, A B-spline Galerkin scheme for calculating the hydroelastic response of a very large floating structure in waves. J. Mar. Science Tech. 3 (1998) 37-49. 